\title{
Lung Function In Children With Scoliosis and Enhanced Spirometry Sensitivity When Using Arm Span Index for Restriction Detection
}

\author{
Snezhina Lazova ${ }^{1}$, Vasil Yablanski ${ }^{2}$,Evgeni Vlaev ${ }^{2}$, Stamatios Priftis $^{3}$, Emilia Naseva $^{3}$, Penka Perenovska \\ and Guergana Petrova ${ }^{1^{*}}$
}

${ }^{1}$ Pediatric Department, University Hospital Alexandrovska, Pediatric Clinic, Medical University Sofia, Bulgaria

${ }^{2}$ Orthopedics Department, Agibadem City Clinic Tokuda Hospital, Bulgaria

${ }^{3}$ Faculty of Public health, Medical University Sofia, Bulgaria

\begin{abstract}
Background: Scoliosis is the most common abnormality of the spine with direct effects on the thoracic cage, leading to potentially severe and irreversible changes in lung function. Scoliosis has generally been associated with the development of restrictive lung disease. Due to the lateral curvature of the spine in children with scoliosis, the measured height does not correspond to the actual one and could not be used as the authoritative index for the calculation of the predicted values for both static and dynamic lung volumes. The indicator arm span (distance between the middle fingers on the outstretched arms horizontally) is a standardized method for calculation of a predictive height in cases where it is impossible to measure the actual one.
\end{abstract}

Aim: To outline the lung function results and to evaluate the utility of arm-span indicator in interpretation of pulmonary function tests (PFT) data of children with scoliosis.

\begin{abstract}
Matherial and methods: For a period of 15 months (2016-2017) we performed pulmonary function tests (spirometry and plethysmography) in 46 children with idiopathic scoliosis aged 5 to 18 years. Anthropometric data was obtained using calibrated stadiometers. The indicator arm span was calculated using Armspan-to-Height software. We compared the predicted height with the measured one and tested the significance of the two values for interpretation of PFT indices.

Results: According to arm span recalculation, the predicted height was higher than the real measured on average by 3.98 $\pm 2.57 \mathrm{~cm}$, which reflected in the number of FEV1\%pred. an average of about $3.9 \% \pm 2.6 \%$ (in some children up to $10 \%$ mismatch) and more significantly for FVC\%pred. an averageof $4.27 \% \pm 2.8 \%$ (up to $>11 \%$ ). This affects the interpretation of PFT - when using the measured height for predictive values some early deterioration in the lung function are missed due to seemingly normal PFT values.

Conclusion: The sensitivity of spiromety as a screening method for the determination of early restrictive changes in vital capacity in children with scoliosis, increases substantially when using arm span to determine the predictive height and comes close to the results from bodypletismography - the gold standard in patients with restriction. Our results may serve as valid justification for introducing the measurement of arm span, instead of actual measuremed height, in patients with diseases with spine deformity like scoliosis, neuromuscular diseases and myopathies.
\end{abstract}

\section{Keywords}

Scoliosis, Children, Spirometry, Arm span, Restriction

\section{Introduction}

Scoliosis is the most common spine deformity with an incidence of 0.3 to $15.3 \%$. According to Scoliosis Research Society (SRS) scoliosis is defined as a lateral curvature of the spine greater than $10^{\circ}$ as measured by the method of Cobb on X-ray radiographs in the upright position [1]. Scoliosis occurs most commonly in periods of rapid somatic growth. In $85 \%$ of cases is an isolated anomaly, which is not associated with any other underlying disease - the so-called idiopathic
*Corresponding author: Dr. Guergana Petrova, Pediatric department, University Hospital Alexandrovska, Pediatric clinic; Medical University Sofia, Bulgaria

Accepted: January 17, 2019

Published online: January 19, 2019

Citation: Lazova S, Yablanski V, Vlaev E, et al. (2019) Lung Function In Children With Scoliosis and Enhanced Spirometry Sensitivity When Using Arm Span Index for Restriction Detection. Ann Pulmonol 3(1):33-38 
Citation: Lazova S, Yablanski V, Vlaev E, et al. (2019) Lung Function In Children With Scoliosis and Enhanced Spirometry Sensitivity When Using Arm Span Index for Restriction Detection. Ann Pulmonol 3(1):33-38

scoliosis. Once the deformity appears it is irreversible, but can be controlled [2]. It is therefore necessary to start a rutine early screening until the skeletal maturity is reached. There is an increasing interest in the relationship between scoliosis and pulmonary function due to the potentially high rates of morbidity and mortality when the disease progresses, which results in decreasing pulmonary function (measured by spirometry and pletismography) [3]. Regular examination of pulmonary function in children with scoliosis can provide appropriate indicators for severity assessment and monitoring. Once documented, the progression of scoliosis needs to be addressed to arrest thoracic cage deformity and concomitant pulmonary compromise [4]. Since poor pulmonary function may lead to a higher incidence of postoperative pulmonary complications, preoperative pulmonary function tests (PFT) have commonly been used to predict postoperative pulmonary complications [5].

A well-known fact is that all results from the spirometry evaluation are calculated as a percentage of the predicted value from the reference equations, which include as a major determinant the height [6]. Thus, any spinal distortion, which leads to a reduction of the real height of patients results in lower predictive values. To overcome this obstacle, we calculated a predictive height based on the arm span indicator (defined as the greatest distance measured between the fingers of both the hands outstretched in horizontal plane).

Aim of our study was to evaluate the preoperative lung fucntion (through spirometry and bodyplethismography) in children with scoliosis and to test the results when calculated according the actual height and the heigh calculated acordingthe arm-span. We aksed ourselves weather using arm-span correction would increase the utilty of spirometry for adequate pre-operative screening method for children with scoliosis.

\section{Matherial and Methods}

For a period of 15 months (2016-2017) we performed PFT (including spirometry and plethysmography), in 46 children with idiopathic scoliosis aged 5 to 18 years. We included all patients with scoliosis referred to orthopaedic department for probable surgical intervention for the mentioned period. We also analysed the PFT results of 41 children without spinal or rib cage deformities - 21 healthy and 20 with bronchial asthma. The healthy controls and asthma patients were chosen to match in age and gender distribution to the scoliosis group. All children in asthma group were with confirmed asthma by pediatric pulmonologist and are followed closely in the clinic.

In all children, included in the study we measured height, weight and calculated BMI. Measurement of height was done with stadiometer using a standardized methodology - while the patient was standing up with the head in the so-called Frankfort horizontal plane with an accuracy of one decimal unit. All patients were barefooted with legs fully attached to the limiter of the stadiometer [7]. The weight measurement is done with a calibrated medical scale.

In children with scoliosis in addition to height in an upright position we measured the distance between the middle fingers on both hands outstretched in horizontal plane - "arm span". The indicator arm span gives information about real height in patients, not for the actual, "shortened" due to spinal distortion. The predicted real height was calculated using Armspan-to-Height software [8] according to their age, gender and ethnicity. We compared the predicted height with the measured one and tested the significance of the two values for interpretation of PFT indices.

In all children with scoliosis on the basis of the conducted imaging studies we defined the type of scoliosis thoracicone, thoraco-lumbar one and lumbar one, as well as the Lenke type and the degree of vertebral rotation were determined. The severity of scoliosis according to Cobb's angle as well as the form of the scoliosis - infantile, adolescent, juvenile was also noted [9]. PFT included forced spirometry, according the ERS/ATS guidelines [10] done with Portable Ultrasonic Diagnostic Plus an incentive spirometer (One Easy spirometer NDD Medical Technologies ${ }^{\circledR}$ ) and body plethysmograph test (Master Screen Body/Diff Jager, Care Germany, Fusion ${ }^{\circledR}$ ). We accepted the tests of all children that meet international quality criteria $[5,10]$.

Informed consent was obtained of all parents for inclusion of the data of their children (anonymized as per GDPR) in this study. The study was approved by Local Ethical Board.

Statistical analysis of the raw data was carried out with program Software package for statistical analysis (SPSS ${ }^{\circledR}$ ), IBM, 2009 version 19 (2010) and Excel (v. 2010). Graphic images representing statistics are prepared mainly with the help of SPSS v.19 and Excel.

\section{Results}

The demographic characteristics of the three groups and their average performance of PFT are presented in Table 1 (the statistical methods used were ANOVA, KruskalWallis). From the 46 children with scoliosis 42 , performed successfully the spirometry and 30 of them could perform the technically correct the body pletismography test. All 41 children enrolled as a control group have spirometry results and bodipletismography that meet international quality criteria $[5,10]$. The three groups have a similar age $(p=0.079)$, gender distribution $(p=0.19$ ) and forced vital capacity (FVC)/ forced expiratory volume for one second (FEV1) ratio ( $p=$ 0.403). The results for FVC\%pred, FEV1\%pred and Maximal mid-expiratory flow 25/75\% (MMEF $25 / 75 \%$ pred) were significantly different with $p=0.009, p=0.002$ and $p=0.001$ respectively. The lowest FVC\%pred was in the scoliosis group, while the healthy and asthmatic children had comparable higher values. The children with asthma demonstrated the lowest MMEF 25/75\%, while children with scoliosis had higher values than asthmatics but lower than the healthy children. The FEV1\%pred was higher in the healthy group while children with scoliosis and children with asthma had comparable lower values (Table 1).

In the group of children with scoliosis we found differences in the normal distribution according the location of the spinal deformity with prevalirane of children with thoraco- 
Citation: Lazova S, Yablanski V, Vlaev E, et al. (2019) Lung Function In Children With Scoliosis and Enhanced Spirometry Sensitivity When Using Arm Span Index for Restriction Detection. Ann Pulmonol 3(1):33-38

Table 1: Demography characteristics and mean PFT results of the patients.

\begin{tabular}{|l|l|l|l|}
\hline Indicator & Scoliosis $\mathbf{( n = 4 6 )}$ & Asthma $(\mathbf{n = 2 0 )}$ & Healthy controls (n-21) \\
\hline Age, years & $14.4 \pm 0.9$ & $13.6 \pm 0.9$ & $13.9 \pm 0.7$ \\
\hline Gender, m:f & $3: 7$ & $4: 6$ & $3: 7$ \\
\hline BMI (mean \pm SD) & $19.4 \pm 0.7$ & $20.9 \pm 1.2$ & $18.5 \pm 1.4$ \\
\hline FEV1/FVC (mean \pm SD) & $87.8 \% \pm 2.9 \%$ & $88.4 \% \pm 2.6 \%$ & $92.3 \% \pm 1.9 \%$ \\
\hline FVC\% predicted & $81.8 \% \pm 3.4 \%$ & $92.1 \% \pm 4.9 \%$ & $100.8 \% \pm 2.9 \%$ \\
\hline FEV1\% predicted & $79.5 \% \pm 4.6 \%$ & $82.3 \% \pm 5.8 \%$ & $105.0 \% \pm 3.5 \%$ \\
\hline MMEF25/75\% predicted & $74.2 \% \pm 9.4 \%$ & $51.5 \% \pm 7.1 \%$ & $115.3 \% \pm 8.8 \%$ \\
\hline
\end{tabular}

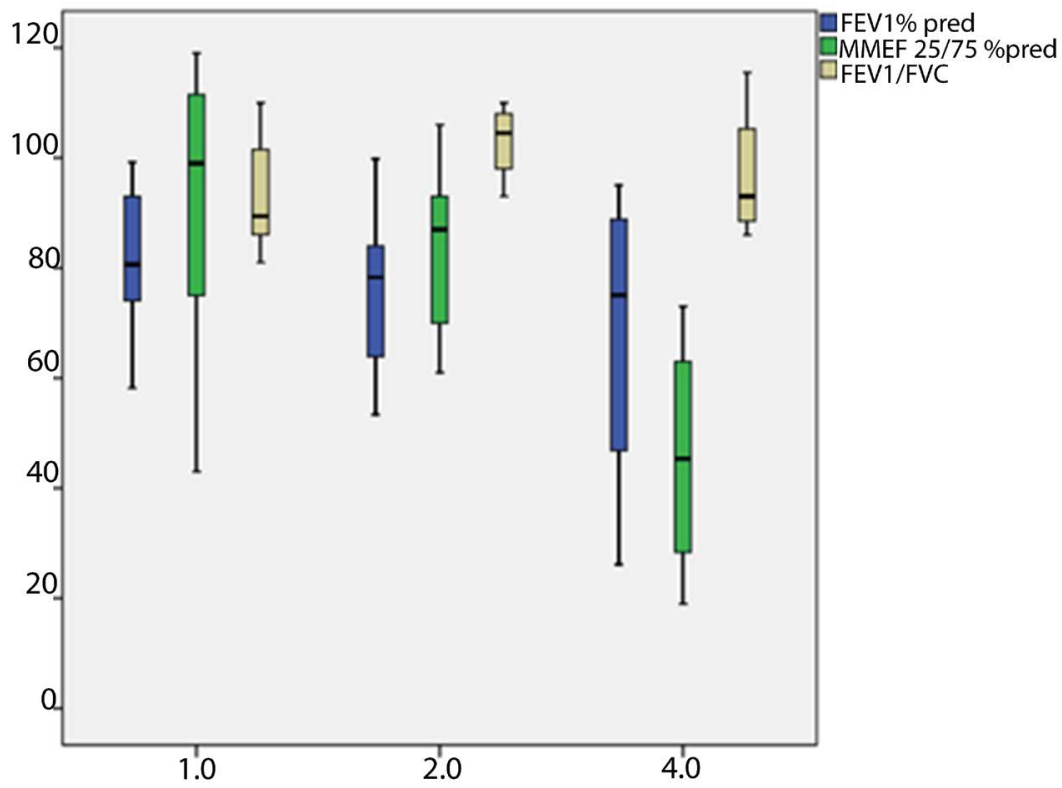

Figure 1: Combined box-plot for FEV1\% pred (blue), MMEF25/75\% pred (green) and Tifno index (beige) In the three forms of scoliosis ( 1 - adolescent one, 2- juvenile one and 4 - infantile one).

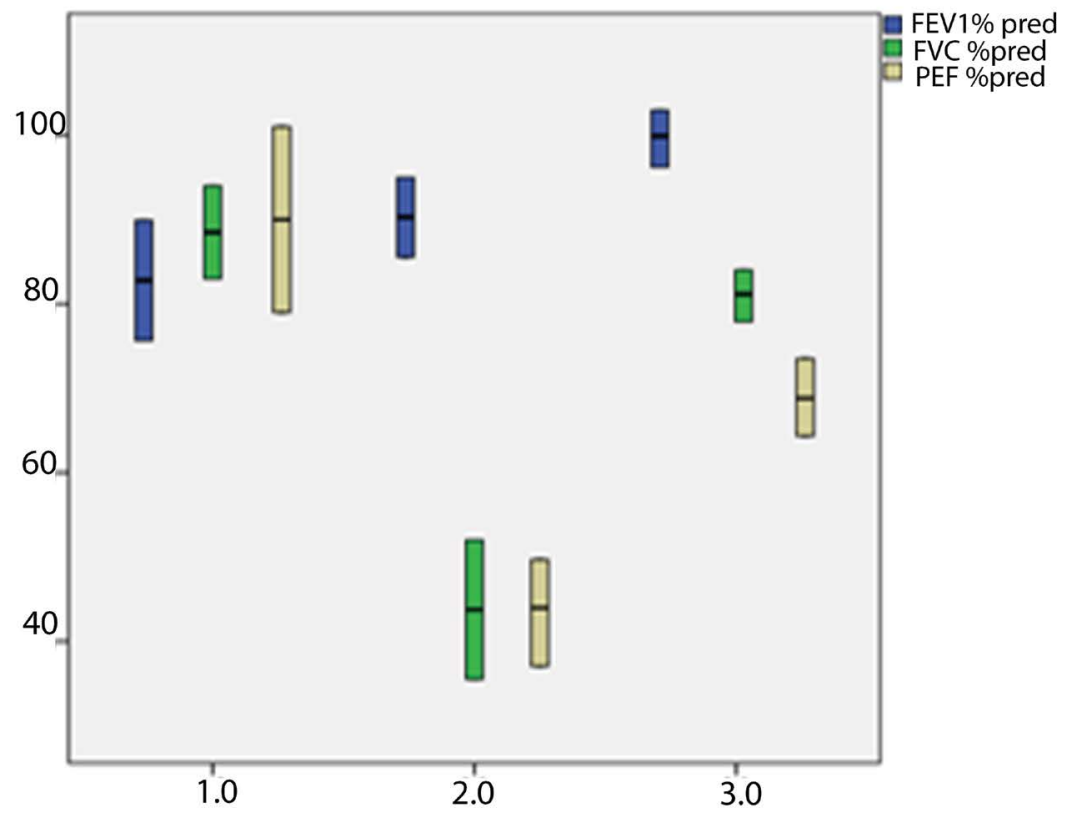

Figure 2: Combined box-plot for FEV1\% pred (blue), FVC\% pred (green) and PEF (beige) In the three forms of scoliosis (1 - thoracolumbar one, 2- thoracic one and 3 - lumbar one). 
lumbar scoliosis vs. the children with either only lumbar or only thoracic one ( $p<0.05$ Chi-square, one sample). From the children with succsessfuly performed spirometry 22 had adolescent scoliosis, 12 - juvenile one and 8 - infantile. We found a statistically significant differences between patients with adolescents, juvenile and infantile forms in terms of Tifno index (FEV1/FVC) $p=0.027$ (ANOVA, post-hoc), FEV1\%pred $p=0.035$ (ANOVA, post-hoc)-75\% MMEF25 pred $p=0.017$ (ANOVA, post-hoc). For the FEV1\%pred and MMEF25 pred$75 \%$ indeces the patients with infantile form showed the lowest values, while the values of those with adolescent one the highest. However, the Tifno index was highest in patients with juvenile scoliosisand lowest in those with adolescent form (Figure 1).

When we looked the indeces according the location of the spinal deformity 27 children were with thoraco-lumbar scoliosis, 11 with only thoracic and 8 with a lumbar scoliosis. Dividing them into three groups we found that statistically significant differences between patients in terms of FVC\%pred $p=0.004$ (ANOVA, post-hoc), FEV1\%pred $p=0.027$ (ANOVA, post-hoc) PEF\%pred $p=0.05$ (ANOVA, post-hoc). The highest PEF and FVC were noted in children with thoraco-lumbar scoliosis, and lowest in those with only thoracic scoliosis. The highest FEV1\%pred was noted in children with lumbar scoliosis while those with thoraco-lumbar one had the lowest score (Figure 2).

The distribution according theLenke type of scoliosis was as follow is: 6 children were with type 1, 6 with type 2, 22 with type 3 , and 3 in each group from type 4 , type 5 and type 6. Dividing them into groups according Lenke type we found statistically significant differences in terms of the difference in FEV1 pre- and post bronchodilator - $\triangle \mathrm{FEV} 1, \mathrm{p}=0.027$ (ANOVA,

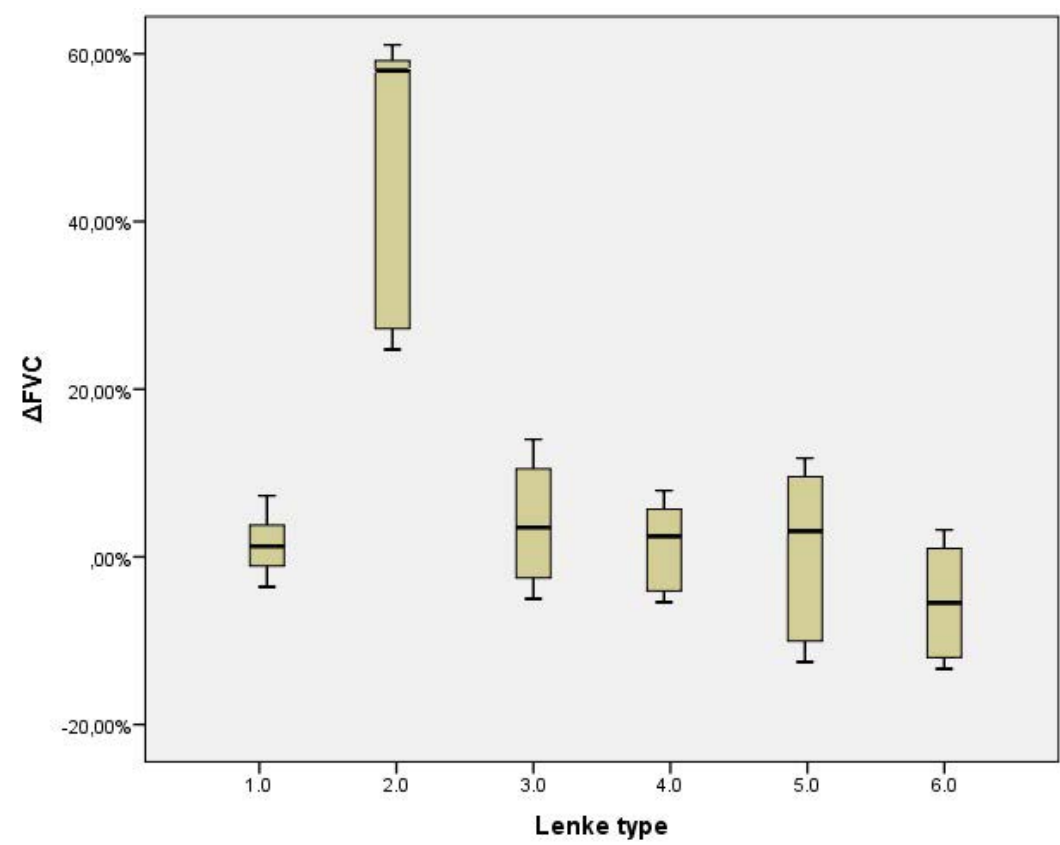

Figure 3: Box-plot for $\triangle \mathrm{FEV} 1$ according the Lenke type of scoliosis.

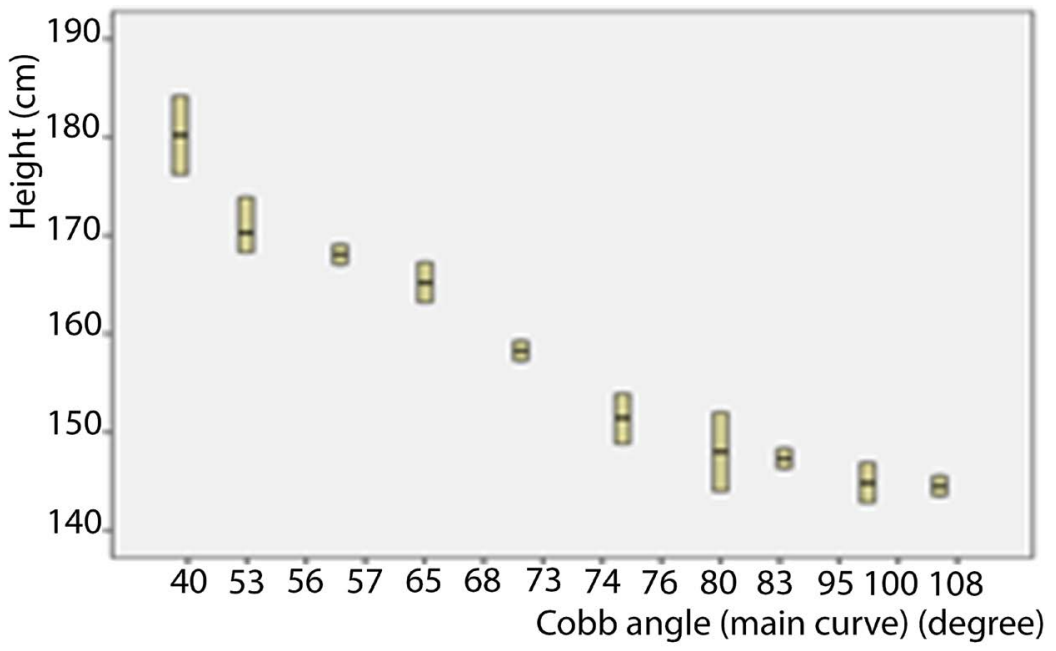

Figure 4: Box-plot for height distribution according the Cobb's angle. 
Citation: Lazova S, Yablanski V, Vlaev E, et al. (2019) Lung Function In Children With Scoliosis and Enhanced Spirometry Sensitivity When Using Arm Span Index for Restriction Detection. Ann Pulmonol 3(1):33-38

post-hoc). The patients with the $2^{\text {nd }}$ type demonstrated the highest positive change in the contrast of patients with types 5 and 6, which demonstrate mostly negative change (Figure 3).

Eleven children had Cobb's angle under $60^{\circ}, 22$ had angle bigger than $60^{\circ}$ and less than or equal to $80^{\circ}$, wit 11 had more than $80^{\circ}$ and less than or equal to $100^{\circ}$, and 2 children had Cobb's angle above $100^{\circ}$. Statisticaly significant differences were found only according the height - which is lower in those with the greatest angle of deformation ( $p=0.30$, ANOVA) without significant changes in other indeces (Figure 4).

Considering that all spirometry indices are calculated as a percentage of the predicted value by reference equations, including the patient's height as the main determinant, and the fact that spinal curvature reduces the actual height of the patients, we recalculated the predicted growth height determined by the largest measured distance between fingers of arms spread in the horizontal span.

We found that, according to arm span recalculation, the predicted height was higher than the real measured on average by $3.98 \pm 2.57 \mathrm{~cm}$, which reflected in the number of FEV1\%pred. an average of about $3.9 \% \pm 2.6 \%$ (in some children up to $10 \%$ mismatch). For the FVC index, the predicted deviation is even more significant - on average by about $4.27 \% \pm 2.8 \%$ (in some children up to more than $11 \%$ difference).

If the height result is not recalculated according the predicted height (as it would have been without the spinal deformity) a damaged lung function could be underestimated - for example in child with FVC\%pred less than $60 \%$ is classified as one with severely diminished lung function, while only $4 \%$ more (when calculated according the measured height) will put him into the moderately-severe lung function affection, similarly if the results are borderline around $80 \%$ the child could be wither with normal wither with mildly diminished lung function. The changes in lung function should be noted as early as possible to prevent the rapid progressive decrease if we don't intervine - classifing the patients as ones with normal function.

When we evaluated the spirometry results according the measured height we found:

- 8 childern have normal lung function(FVC $\geq 100 \%$ )

- 26 children have mildly affecteed lung function (FVC $\geq$ $80 \%$ and $<100 \%$ )

- 8 children have moderately-severe lund function disturbance (FVC $\geq 60 \%$ and $<80 \%$ )

- 4 children have severely diminished lung function (FVC $<60 \%$, and only one is very severely affected with FVC < 40\%)

When we recalculated the height (according to the measured value for the arm span), however, we found that:

- Only one child has normal lung function FVC $\geq 100 \%$ ( $p=$ 0.025 Chi-square)

- 21 children have mildly affecteed lung function ( $p=0.052$ Chi-square)
- 14 children have moderately-severe lund function disturbance ( $p=0.24$ Chi-square)

- $\mathbf{1 0}$ children have severely diminished lung function $(p=$ 0.09 Chi-square).

Therefore, using the real-measured height to determine the predicted spirometry index values in children with scoliosis, instead of the predicted one (using surrogate measures like arm span), early pulmonary function abnormalities could possibly remain undetected, when the calculated indices based on the measured height only have seemingly normal values.

The results we obtained are convincing and are a strong ground for using the arm span calculated height instead of the real-measured one when performing lung function tests in pulmonology practices for children with idiopathic scoliosis or scoliosis as a result of neuromuscular diseases and myopathies.

\section{Discussion}

Scoliosis is a common deformity affecting the thoracic cage, with potentially severe and irreversible effects on lung function. Most commonly it occurs in periods of rapid somatic growth. Characteristic of patients with scoliosis is the development of restrictive ventilatory defect (proportionally reducing all lung volumes) [1,3,6,11-14].

Characteristic for patients with scoliosis is the development of a restriction (proportional reduction of all lung volumes) and especially reduced total lung capacity (TLC). Expiratory debits are proportionaly reduced to the restricitve lung volumes, while the ration FEV1/FVC remains in the normal range. The reduction in the lung volumes is result from multiple factors. It is determined by the angle of the scoliosis (Cobb angle $>70^{\circ}$ ), the numbers affected vertebrae ( 7 or more) the exact location on the curvature and the loss of the normal thoracic kyphosis [15-17]. Nevertheless the primary reason for the restriction, prolonged hipoinflaciâ and atelectasis can potentially lead to an irreversible atrophy and further reduction in lung volume. The reduction of TLC is often associated with increased residual volume (RV), leading to an increased RV/TLC ratio. The latter is a commonly used as an index for air retention and is usually seen in patients with obstructive lung disease (severe asthma, cystic fibrosis, etc). In patients with scoliosis hiperinflacion can be present even if there is no clicnical of PFT data for airwayobstruction. This could be probably due to the dysfunction of the expiratory muscles and inability to reach full exhalatio. In these cases due to increased RV, FVC and the total inspiratory capacity are often more severly reduced from the expected value based on TLC [18].

Measurement of TLC requires specialized equipment (bodipletizmography or Nitrogen washout test). In most cases, spirometry can also provide good and reliable indicators for the assessment of the existence and severity of the restrictive defect. The reduction of the FVC, measured with spirometry, is proportional to the reduction of TLC, except in the case of obstructive or mixed ventilatory defect $[17,19,20]$. 
Citation: Lazova S, Yablanski V, Vlaev E, et al. (2019) Lung Function In Children With Scoliosis and Enhanced Spirometry Sensitivity When Using Arm Span Index for Restriction Detection. Ann Pulmonol 3(1):33-38

The airways in patients with mild to moderate scoliosis remain unaffected. In more severe cases a characteristic form of the expiratory part of debit-volume curve is observed high and narrow with with maximum expiratory flow rates, increased disproportionately in relation to lung volumes (thus reflectting the rapid emptying of the lung). Inspiratory part of the debit-volume curve is a mirror image of the expiratory part. With progression and deterioration of the situation, it is possible a concavity in the expiratory loop of debitvolume curve to be observed, as a proof of the existence of a concomitant lower airway obstruction [13].

It is very important to note that in children with scoliosis height cannot be used as a benchmark for the calculation of the values for the static and dynamic volumes. Methods that can be used as a surrogate of the height are the measurement of arm span or the length of certain long bones. This is confirmed also by our results, as it follows: with the use of actual measured height to determine the values of the predictive values of the PFT indices in the interpretation of spirometry in children with scoliosis, instead of the recalculated one, leads to underestimation of the early decrease the lung-function indicators, while they seem normal when calculated according to measured height.

The sensitivity of spirometry as a screening method for determining vital capacity disorders and detection of initial restriction in children with scoliosis is significantly increased when using arm span to determine the height. The sensitivity of arm-span height-adjusted spirometry is approaching that of plethysmography, which is considered as a gold standard in patients with restriction Spirometry is the most accessible and widely-used lung function test in medical practice for it is well-standardized, non-costly and doesn't require a speciallyequipped functional laboratory. Arm span indicator and height recalculation will enhance spirometry's validity and sensitivity in the comprehensive approach (follow up and treatment) protocol in children with vertebral deformities. This method is practically easy to apply and could have a significant medical (biological, psychological) and social impact.

\section{Acknowledgements}

This work was supported by a grant from the Medical University of Sofia (Council of Medical Science, project no. $513 / 2$.

\section{References}

1. Society SR (2009) Adolescent Idiopathic Scoliosis.

2. Stirling AJ, Howel D, Millner PA, et al. (1996) Late-onset idiopathic scoliosis in children six to fourteen years old. A cross-sectional prevalence study. J Bone Joint Surg Am 9: 1330-1336.
3. Johari J, Sharifudin MA, Ab Rahman A, et al. (2016) Relationship between pulmonary function and degree of spinal deformity, location of apical vertebrae and age among adolescent idiopathic scoliosis patients. Singapore Med J 57: 33-38.

4. Newton PO, Faro FD, Gollogly S, et al. (2005) Results of preoperative pulmonary function testing of adolescents with idiopathic scoliosis. A study of six hundred and thirty-one patients. J Bone Joint Surg Am 87: 1937-1946.

5. Pellegrino R, Viegi G, Brusasco V, et al. (2005) Interpretative strategies for lung function tests. European Respiratory Journal 26: 948-968.

6. Zhang JG, Wang W, Qiu GX, et al. (2005) The role of preoperative pulmonary function tests in the surgical treatment of scoliosis. Spine (Phila Pa 1976) 30: 218-221.

7. (2003) Training manual for height and weight assessment originally developed by BMI task force.

8. http://spirxpert.ers-education.org/en/download/armspan-toheight-software/

9. Lonstein JE (1994) Adolescent idiopathic scoliosis. Lancet 344: 1407-1412.

10. (2005) ATS/ERS task force: Standardisation of lung function testing. Standardisation of spirometry. Eur Respir J 26: 319-338.

11. Koumbourlis AC (2006) Scoliosis and the respiratory system. Paediatr Respir Rev 7: 152-160.

12. Boyer J, Amin N, Taddonio R, et al. (1996) Evidence of airway obstruction in children with idiopathic scoliosis. Chest 109: 1532-1535.

13. Day GA, Upadhyay SS, Ho EK, et al. (1994) Pulmonary functions in congenital scoliosis. Spine (Phila Pa 1976) 19: 1027-1031.

14. Barrios C, Pérez-Encinas C, Maruenda JI, et al. (2005) Significant ventilator functional restriction in adolescents with mild or moderate scoliosis during maximal exercise tolerance test. Spine 30: 1610-1615.

15. Kearon C, Viviani GR, Kirkley A, et al. (1993) Factors determining pulmonary function in adolescent idiopathic thoracic scoliosis. Am Rev Respir Dis 1993: 288-294.

16. McMaster MJ, Glasby MA, Singh $\mathrm{H}$, et al. (2007) Lung function in congenital kyphosis and kyphoscoliosis. J Spinal Disord Tech 20: 203-208.

17. Tsiligiannis T, Grivas T (2012) Pulmonary function in children with idiopathic scoliosis. Scoliosis 23: 7.

18. Cooper DM, Rojas JV, Mellins RB, et al. (1984) Respiratory mechanics in adolescents with idiopathic scoliosis. Am Rev Respir Dis 130: 16-22.

19. Aaron SD, Dales RE, Cardinal P (1999) How accurate is spirometry at predicting restrictive pulmonary impairment? Chest 115: 869-873.

20. Venkateshiah SB, loachimescu OC, McCarthy K, et al. (2008) The utility of spirometry in diagnosing pulmonary restriction. Lung 186: $19-25$.

DOI: $10.36959 / 918 / 449$

Copyright: (c) 2019 Lazova S, et al. This is an open-access article distributed under the terms of the Creative Commons Attribution License, which permits unrestricted use, distribution, and reproduction in any medium, provided the original author and source are credited. 